\title{
ARCHITECTURE AND DESIGN OF AN INTEGRATED WEARABLE, IOT AND CLOUD BASED TELEMEDICINE SYSTEM FOR RURAL HEALTHCARE
}

\author{
Vanita Ganesh Bhagwat ${ }^{1}$, Muzameel Ahmed ${ }^{2}$, Arbind Gupta ${ }^{3}$, Sreevani $\mathbf{N}^{4}$ \\ ${ }^{I}$ Student, Department of ISE, DSCE, Bangalore \\ ${ }^{2}$ Research Scholar, Jain University, Assistant Professor, Department of ISE, DSCE, Bangalore \\ ${ }^{3}$ Professor, Department of CSE, DSCE, Bangalore \\ ${ }^{4}$ Software Consultant, Bangalore
}

\begin{abstract}
With, more than $70 \%$ of the population living in rural parts of India, availability of health-care in these parts of the country is largely constrained by shortage of medical professionals, reluctance of doctors to work in rural areas, unavailability of basic medical facilities and functionaries etc. A Telemedicine system can help in bridging this gap by making use of furtherance in sensor technology, information technology and telecommunication technology to deliver the healthcare remotely. But the available have not found much acceptance in rural areas due to various reasons such as High cost barrier, Dependency on grid electricity, Maintenance challenge, unavailability of native language support etc. Objective of this paper is to design a telemedicine architecture that is suitable to be used in rural parts of the country.
\end{abstract}

Keywords: - Telemedicine, health-care, Remote health, Tele-Consultation, IoT, Cloud

\section{INTRODUCTION}

Dearth of pertinent information at correct time and place has a great effect on health-care principally in the case of developing countries like India where availability of healthcare is constrained by affordability, lack of awareness about the diseases, illiteracy, unavailability of doctors etc. Despite significant effort put by government in creating basic infrastructure of 153655 SCs, 25308 PHCs, and 5396 CHC [1] health-care is not universally available.

In India, there are only 0.7 doctors for every 1000 people compared to - Russia-(5), USA-(2.3), Brazil-(1.5) and China-(1.5) [2]. In addition, there is reluctance of specialists and quality doctors to serve rural population and inadequate facilities in health centers. On an average, a person travels about $8 \mathrm{KM}$ for accessing basic health facility which puts a significant barrier for pregnant women, babies and children. In addition to this, there is lack of awareness about health issues and an ignorance of proper healthcare in rural population.

Several organizations are working alongside the government and NGOs to make healthcare accessible everywhere. The government has created a good physical infrastructure of PHCs / PHU / CHC across India but, there is a large gap in availability and need of doctors in rural parts of the country. People cannot afford to go to hospitals in bigger cities, nor can we make hospitals and doctors work in rural areas. A telemedicine system can help in bridging this gap but available systems are not affordable enough given the socio- economic conditions of rural populationand it requires trained staff or doctors to use it. Many telemedicine solutions such as KTwo Kshema, eVaidya, CDAC, ISRO, Apollo, etc exist. But they have not found much acceptance in rural India due to

(a) High cost a barrier to adoption (eg KTwo system costs $7+$ lakhs);

(b) Dependency on grid electricity for operation;

(c) Maintenance challenge due to larger number of components and higher rate of failure due to wired connectivity;

(d) Complex user interface;

(e) Lack of native language support etc.

One of the cornerstones of the Universal Health Coverage (UHC) initiative enunciated by W.H.O. is use of integrated health technologies and medical devices which are available today. Availability of low cost $4 \mathrm{G}$ phones, tablets, Digital India infrastructure, biosensors etc, make it possible to deliver healthcare that is available for everyone, everywhere and every day.

A better solution which is in line with the requirements of rural population can be delineated by using bottom up design approach. Emergence of new technologies and easy availability of varied low-cost hardware platform make it possible to reduce the cost associated with developing and maintaining the system there by making the system affordable and more suitable for Rural scenarios. 
Proposed is the architecture for telemedicine system with layered architecture, where each layer has a specific and well-defined role. It makes the system extensible and easy to manage.System is designed as a composition of RESTful web services over HTTP.

\section{LITERATURE SURVEY}

Individual components of solutions (sensors, network, mobile hand-held devices, wireless connectivity) already exist.

In India works linked to Telemedicine were initiated in 1999. Since that year, ISRO has set up a Satellite Communication-(SATCOM) based telemedicine systems around the country. Many government agencies like Department of Information Technology and Ministry of Health \& Family Welfare, state governments, premier medical and technical institutions of India have initiated work towards telemedicine with the intent of presenting quality healthcare facilities to the rural and remote parts of the country [3]. The Government of India has proposed and carried out various national-level projects and also extended telemedicine services to South Asian and African countries [3].

TeleVital's presence in the American and Indian markets spans over a period of 10 years, and has implemented more than 515 Telemedicine installations throughout the world. In India, TeleVital is the preferred Telemedicine technology solution provider to the Indian Space Research Organization (ISRO) sponsored projects.

Presently, ISRO's Telemedicine Network stretches to around 100 Hospitals all over the country and has treated more than 25000 patients. They provide solution for point to point system and multipoint to multipoint system. Both use satellite communication for connecting patient side device, at a remote location, to a remote doctor.

Telemedicine system used for detection, monitoring and treatment of commonly seen diseases is established by DITDepartment of Information Technology, MCIT-Ministry of Communication and IT in association with Government of India in West Bengal [3]. They haveestablished more than 75 nodes all over the India. Project uses ISDN, WAN \& Satellite as a means for connection establishment[3].

Amrita center for digital health provides telemedicine solution by linking various clinics and hospitals located in the remote areas of India with Amrita Institute of Medical Sciences and Research Centre. This aims to provide the best medical care to people in remote corners of India, who cannot afford the treatment due to their inability to travel, cost, unawareness etc. This is being provided at more than 60 centers in India that are connected from AIMS.
Government of different states (Uttar Pradesh, Orissa, Chhattisgarh, Kerala, Andhra Pradesh) have made progress in the direction of connecting its sub centers or secondary health centers(SC) to medical collegesand multi-specialty hospitals for providing care remotely [3]. State Government of Rajasthan and Karnataka in partnership with ISRO, have set up telemedicine networks for making healthcare available in rural parts of the states[3].

The state-of-art telecommunication network has mainly focused on four verticals TB, Kala-azar, childhood diarrhea and pneumonia. An MoU was signed in 2011 between the state government and Bill and Melinda Gates (BMG) foundation to strengthen health care projects for the state. A patient from his/her own village or nearby village through SHC are diagnosed by a panel of specialists sitting at central medical facility in Delhi, Patna, local area doctor with the help of modern technologies like internet, computer, web cameras and other devices. Each SHC village is responsible for about 6-7 catchment villages. In every catchment village, the SHC franchisee identifies woman health provider with her male counterpart (rural health provider) or ASHA workers. The organization provides training to the selected couples and brands them as Sky Care, where cellphone consultation facilities are available.

Telemedicine application named Sanjeevani, launched in 2009 by union IT minister, has been upgraded to a web based application e-Sanjeevani (using .Net Technology). eSanjeevani is a result of core $R \& D$ effort in the area of Healthcare, Medical Informatics at C-DAC Mohali, India, developed state-of-art telemedicine technology software package named "Sanjeevani" in-house They also established telemedicine sites at different locations in the state of Himachal Pradesh and also working on setting it in rural areas of Punjab.

Tele-Consultation: eSanjeevani provides tele-consultation facility using both 'store and forward' and 'real-time' mode. In a 'store and forward' mode, the users can choose the doctors in the specialised area for the diagnosis and send the selected/ entire EMR for consultation. For 'real-time' consultation, video conferencing facility is provided to the user to connect to the doctor.

Medical Equipment Interface: 'eSanjeevani' provides an interface with a wide range of diagnostic/ medical equipment. Output of each of the medical peripherals is directly acquired by eSanjeevani. Some of the medical diagnostic equipment interfaced with eSanjeevani include ECG machine, digital slit lamp, digital microscope, medical film scanner, etc.

Supposed to have pioneered the concept of telemedicine in India Narayana Hrudayalaya has about 150 telemedicine centers connected. The focus of their telemedicine service is for Cardiac patients and they have treated more than 54000 cardiac patients so far and have 63 installations in Africa and 10 globally. They are partly funded by Hewlett Packard. 
They are making use of ISRO network to connect 322 hospitals and 299 district health centers to 33 multi-specialty hospitals in India [3]. These nodes use telephone line, broadband or satellite for data transfer which is provided by ISRO [3].

Telemedicine project from Apollo Hospitals, has opened telemedicine centers that connect remote villages via satellite to centers that provide professional medical services. Aragonda project from Apollo Hospital was India' s 1st rural medicine station[3].

Srijani Mukherjee et al. [7] proposed a telemonitoring architecture, which is a networked system for monitoring the health remotely. System proposed in this paper makes use of 3 -tier architecture in the form of perception, middleware and service layer [7].

Victor chang et al. [8] talks about multi layered architecture for cloud security in their paper. This security architecture offers real time security and protection to peta bytes of data which is relevant for health-care systems. [8]

To have a system that is convenient for delivery of healthcare to rural areas we need to take following things into consideration.

Cost Involved: For this system to be accepted in rural sector the monetary investment involved in setting up the telemedicine system and providing consultation remotely should be very low. A low-cost solution can also create entrepreneurship opportunities considering the various startup schemes from government of India such as Startup India. Telemedicine systems from ISRO costs approximately 3.5 lacks. Kshema Telemedicine system from K2 global costs approximately 8 lacks. E-Sanjeevini telemedicine system designed by CDAC costs 4 lacks and so on. Therefore, affordability is one of the main reasons why the existing telemedicine systems aren't found extensive approval. Along with the infrastructure provided by the government, A low cost solution can Encourage entrepreneurship for setting up hyper-local health clinic in rural areas.

Seamless transfer of data in areas with low or no network connectivity: It is important for the system to ensure smooth and continues consultation. Telemedicine systems mentioned above have used VSAT, Satellite, Optical Fiber, Broadband, Wireless etc. for data transfer and communication. Some of these are not suitable for rural areas where network connectivity is an issue.

Usability: User interface should be simple and easy to understand with native language support. It should not require extensive training to use the system. It should be designed to meet the needs of primary users of the system (Semi skilled people).
Maintainability: System should be rugged, fault tolerant and should not require much maintenance (or can be easily maintained)

Operational ease: System must be able to work without continuous electric supply and have less components.

\section{USE CASES}

4.1-At one end of the system is the caregiver who registers himself into the system through a UI provided to him.

4.2-Caregiver $\operatorname{logs}$ in to the system and registers the patient with provided personal information of the patient.

4.3-At the other end of the system is a physician who registers himself into the system through the physician portal.

4.4-Information about the time at which the physician is available for consulting the patient is collected.

4.5-Appointment with physician is scheduled by the caregiver after collecting patient's preferences.

4.6-At the time of consultation, the caregiver connects the sensors to a patient and collects his vital parameters such as body temperature, blood sugar level, Oxygen saturation levels(SPO2) etc on physician's request.

Collected, patient data is encrypted transferred to the cloud or central server and from there it is transferred to the physician to view.

4.7-Physician analyzes the current and historical patient data and provides diagnosis or prescription which will transferred back to the patient end.

4.8-All the details related to patient's problem, encounter between the patient and physician, caregiver handling this communication, prescription provided will be stored in central server.

4.9-Real time audio/video communication between the patient and the physician is provided by the system.

4.10-The system facilitates the consultation even in the places where mobile connectivity is unavailable by making use of Software Defined Radio(SDR) communication system or Long-Range Wireless Personal Area Network (LORA WPAN).

\section{PROPOSED FRAMEWORK}

\subsection{Overview}

Below is the Business architecture of the proposed system. 


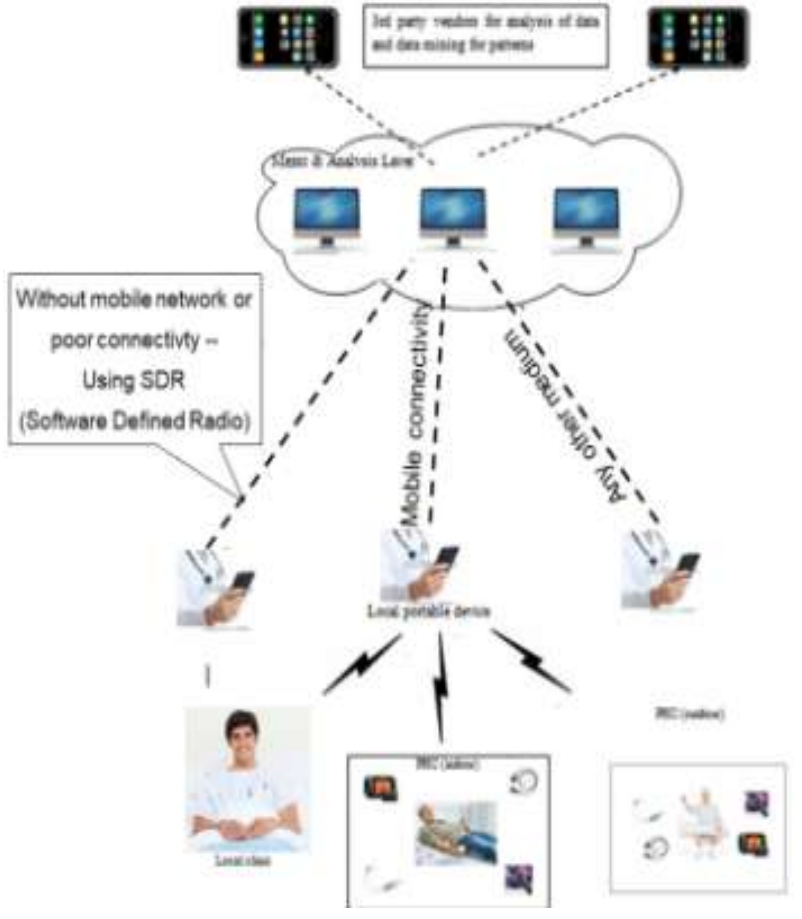

Main external entities of the system include Physician at remote place providing health care, Patient getting treated, Caregiver to operate the system and to assist the patient.

Proposed system follows layered architecture style.It separates the data access layer from business logic layer and business logic layer from presentation layers which enables user interaction with the system.

System can either use Store and forward or two-way interactive television technique based on the type of the information that need to be communicated.

Proposed architecture makes use of Service Oriented Architecture(SOA) to facilitate the interaction between the heterogeneous systems. Using SOA, the business tasks of the system are transfigured into set of webservices that are accessible over the internet. The architecture of the system is realized using Representational State Transfer(REST) architectural style. REST presents web services as URIs which are used as resources to serve the request to the system.

\subsection{Layered Architecture}

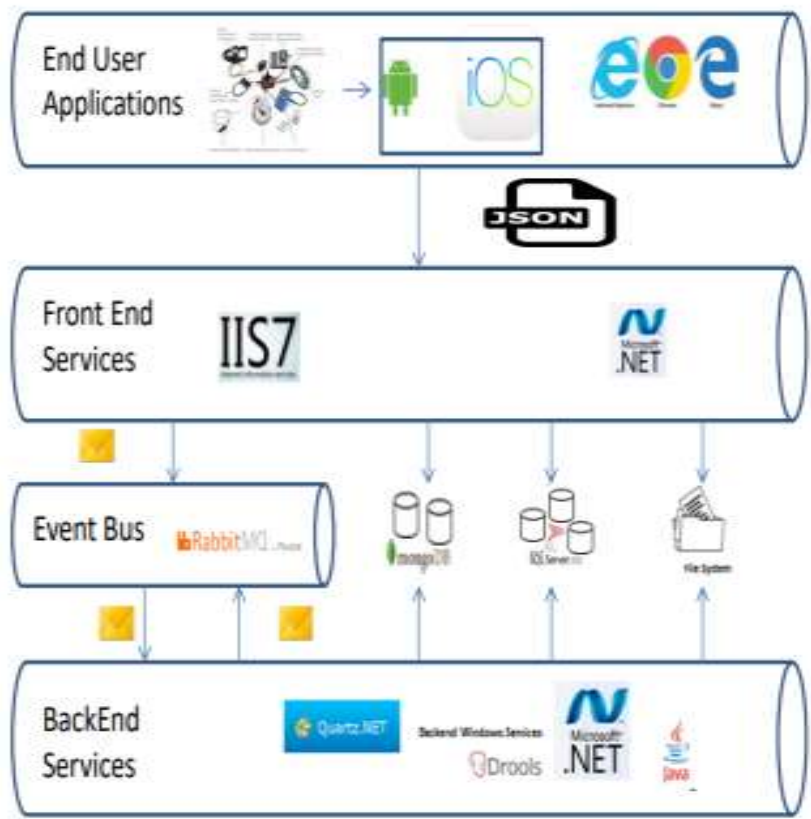

End user applications such as physician portal and caregiver portal are developed as internet applications.

Front end services such as registration, scheduling is developed as REST Web services using Microsoft .NET web API, which can be hosted on IIS web server.

Data collected from the system is stored in different storage means based on the type of the data. Reference data that users do not modify can be stored in file system for faster access. All the structured data such as information related to caregiver, patient and physician can be stored in a relational database system. In our system Microsoft SQL server 2017 is used. Unstructured and semi structured such as video and audio data related to encounters are stored in Non-relational database such as Mongo-DB.

The system makes use of message queue for collecting the data from the occasionally connected clients, making the system asynchronous.

\subsection{Security}

Security is essential part of the healthcare systems because of the nature of the information involved. Personal and medical information of a patient is confidential and critical and hence needs to be protected. A multilayered security architecture can help in maximizing the protection of the personal health information involved.

At first layer of defense is the access control, which can be implemented through authentication and authorization which allows only the authenticated users to enter the system and only the authorized members to have access to information. 
At second layer of security, is detection and prevention of attacks such as DoS, pattern based attacks, trojans etc with the help of Intrusion detection and prevention techniques such as pattern matching algorithms.

The third layer of security can be provided by making use of encryption techniques such as AES, Triple DES, RSA etc.

\section{CONCLUSION}

The proposed is the architecture of telemedicine system which is expected to meet the market needs of the rural population. A low-cost solution can help in getting much wider acceptance and it will also enable and accelerate private deployment of the systems by making use of available entrepreneurship skills in rural areas. It is expected to give high reliability and data integrity because of its asynchronous nature.

Use of the S.O.L.I.D principles make a system easy to maintain, easy to understand, easy to read, easy to change. It also improves the shelf life of the software products.

\section{REFERENCES}

[1] National Health Mission report for 2016-17

[2] Price Water House Coopers

[3] Uzzal Kumar Prodhan, Muhammad ZahidurRahman, Israt Jahan. "Telemedicine in SouthAsia for rural people: Current scenario andfuture recommendations", 2015 InternationalConference on Computer and InformationEngineering (ICCIE), 2015

[4] Mishra, S.K., Basnet, R. and Singh, K. "Current telemedicine infrastructure, network, applications in India," $81 \mathrm{~h}$ IEEE International Conference on ehealth networking, application and services, pp.4649, 2006.

[5] Saroj Kanta Mishra, Indra Pratap Singh and Repu Daman Chand "Current status of telemedicine network in India and future perspective," The proceedings of the Asia-Pacific Advanced Network, vo. 32, pp. 151-163,2012.

[6] Krishnan Ganapathy and Aditi Ravindra "Telemedicine in India: The Apollo story: Apollo telemedicine networking foundation," Telemedicine and e-health, vol. 15, no. 6, july/august 2009.

[7] Srijani Mukherjee, Koustabh Dolui, Soumya Kanti Datta, "Patient Health Management System using eHealth Monitoring Architecture" 2014 IEEE International Advance Computing Conference (IACC).

[8] Chang, Victor, and Muthu Ramachandran. "Towards achieving Data Security with the Cloud Computing Adoption Framework", IEEE Transactions on Services Computing, 2015.

[9] Dr. P. S. Ramkumar1, Balasubramanya Vasista, Chandan Prakash, Sandeep Patil H G, Prasanna Datha. "Cloud based Tele-Monitoring System for PatientCare", International Journal of Engineering
Research \& Technology (IJERT), ISSN: 2278-0181, Vol. 5 Issue 04, April-2016

[10] Martin Krohn, Heiko Koppand Djamshid Tavangarian. "A wireless architecture for telemedicine", 4th Workshop on postponing, navigation and communication 2007 (WPNC'07), Hannover, Germany.

[11] Cornel Popescu. "Autonomous Systems for Telemedicine”, 2015 Ninth International Conference on Complex, Intelligent, and Software Intensive Systems.

[12] S. M. RIAZUL ISLAM, AEHAN KWAK, MD. HUMAUN KABIR, MAHMUD HOSSAIN, AND KYUNG-SUP KWAK. "The Internet of Things for Health Care: A Comprehensive Survey" IEEE Access, date of publication June 1, 2015, date of current version June 4, 2015. 